\title{
Evaluación de neofobia en un rebaño de gamos en cautividad
}

\author{
Iglesias, C. ${ }^{\text {; }}$ Navas, F.J. ${ }^{1}$; Pizarro, G. ${ }^{\text {; }}$ Arando, A. ${ }^{\text {; }}$ Cumplido, A. ${ }^{\text {i }}$; Delgado, J.V. ${ }^{1}$ and Ruíz, M.J. ${ }^{2}$
}

'Department of Genetics. University of Córdoba. Córdoba. Spain.

2Área de Conservación. Parque Zoológico Municipal de Córdoba. Córdoba. Spain.

\section{PALABRAS CLAVE ADICIONALES}

\section{Neofobia.}

Estrés.

Zoológico.

Gamo.

\section{RESUMEN}

La neofobia es un comportamiento ecológicamente relevante que surge a través de una evaluación cognitiva de estímulos novedosos. Evaluamos las respuestas de 8 gamos en cautividad, utilizando una prueba de reacción frente a objetos noveles y una escala de evaluación cualitativa del comportamiento. El análisis de componentes principales categóricos reveló que la variabilidad presente en la población para la prueba de neofobia explicaba el 69,94\% de la varianza encontrada en el rebaño estudiado. Se detectó que las variables estaban desde pobremente correlacionadas hasta altamente correlacionadas, tanto positiva como negativamente. Las correlaciones negativas entre las variables medidas variaron desde -0.005 (entre el tiempo que el animal está distraído y la edad) hasta -0.912 (entre la edad y la jerarquía), y los positivos desde 0.005 (entre el tiempo de aproximación hasta un metro y la edad) hasta 0.831 (entre el tiempo que el animal está distraído y el tiempo que el animal se toma para prestar atención, pero permanece inmóvil). Estos hallazgos resaltan la naturaleza de las respuestas neofóbicas en ungulados bajo cautiverio y cómo la estructura social las afecta.

\section{Evaluating neophobia in a captive herd of fallow deers}

\section{SUMMARY}

\section{ADDITIONAL KEYWORDS}

Neophobia.

Distress.

Zoo.

Fallow deer.

INFORMATION

\section{Cronología del artículo.}

Recibido/Received: 18.06 .2018

Aceptado/Accepted: 01.10.2018

On-line: 15.10 .2018

Correspondencia a los autores/Contact e-mail:

fjng87@hotmail.com
Neophobia is an ecologically relevant behaviour that arises through a cognitive assessment of novel stimuli. We assessed the responses of 8 captive fallow deer, using a novel object test and a qualitative behaviour assessment scale. The categorical principal component analysis revealed that the variability present in the population for the neophobia test explained $69.94 \%$ of the variance found in the herd studied. It was detected that the variables were from poorly correlated to highly correlated, both positively and negatively. The negative correlations between the measured variables varied from -0.005 (between the time the animal is distracted and the age) up to -0.912 (between age and the hierarchy), and the positives from 0.005 (between the time of approximation up to a meter and the age) until 0.831 (Between the time the animal is distracted and the time that the animal takes to pay attention but remains motionless). These findings highlight the nature of neophobic responses in ungulates under captivity and how social structure impacts them.

\section{INTRODUCCIÓN}

La domesticación es el proceso por el cual una determinada especie animal, cuya actividad vital se desarrolla en libertad, adquiere o desarrolla ciertos caracteres fenotípicos y de comportamiento como consecuencia de la selección artificial por parte del ser humano o una selección natural de progresiva adaptación a la convivencia con este (Denis, 2004). Además de constituirse como un fenómeno de notable importancia cultural, la domesticación, en términos biológicos, involucra la concatenación de importantes cambios genéticos producto de la intervención humana y que se suceden a lo largo de las diferentes generaciones animales.
Importantes avances en las tecnologías de secuenciación genética durante las últimas décadas han supuesto un avance en el conocimiento que se tiene sobre el proceso de domesticación animal, al constituir un marco multidisciplinario conceptual que integra ideas derivadas de la evolución biológica como son la epigénesis, plasticidad genética y genética poblacional cuantitativa. Su comprensión es clave para un mejor entendimiento de los efectos de la selección artificial sobre los cambios fenotípicos y la caracterización de su heredabilidad (Gross \& Olsen, 2010; Larson et al., 2014).

Independientemente de si se encuentran en su hábitat natural o en cautividad, el comportamiento ex- 
ploratorio es un acto decisivo para que los animales integren la información que reciben de su entorno y tomen decisiones en base a ella, a la vez que este hecho influye directa o indirectamente en la reproducción y supervivencia de las poblaciones animales (Dall, Giraldeau, Olsson, McNamara, \& Stephens, 2005; MettkeHofmann, Winkler, \& Leisler, 2002). La exploración y evaluación del entorno pueden realizarla los animales de forma autónoma o por efecto del aprendizaje social en grupo (Coolen, Bergen, Day, \& Laland, 2003; Swaney, Kendal, Capon, Brown, \& Laland, 2001).

En términos de adaptación evolutiva, la neofobia o miedo incontrolable e injustificado conscientemente hacia elementos o experiencias nuevas, no solo puede minimizar la exposición al peligro sino también limitar el propio comportamiento de exploración (Stöwe, Bugnyar, Heinrich, \& Kotrschal, 2006; Stöwe, Bugnyar, Loretto, et al., 2006). El interés suscitado por un elemento o experiencia nueva en un animal es el determinante de la magnitud de la respuesta neofóbica, de ahí que una parte de los animales se acerquen de forma rápida a este elemento para explorarlo, mientras que otra parte de los animales tardarán en hacerlo (Coolen et al., 2003) o, incluso, recurrirán a la evitación activa por desinterés o falta de relevancia percibida. En aquellos animales con un temperamento nervioso o excitable, lo nuevo o extraño en su entorno puede actuar al mismo tiempo como elemento atractivo y como elemento que suscita miedo (Grandin \& Deesing, 2014). En función de la ecología de la especie, la motivación del animal y la estructura jerárquico-social de las poblaciones, los individuos explorarán los cambios en su entorno familiar con diferentes latencias y por períodos variables (Sol, Timmermans, \& Lefebvre, 2002; Stöwe, Bugnyar, Heinrich, et al., 2006; Stöwe, Bugnyar, Loretto, et al., 2006).

En concordancia con lo anterior, Schultz (2013) encontró que cuando el rango de estímulos al que un animal es sometido a lo largo de su desarrollo es limitado, la regulación de los umbrales de sensibilidad a nivel central será un proceso desencadenado a niveles más bajos de estimulación. No obstante, los animales se prestarán más interesados ante un estímulo novedoso cuando se les permite acercarse a este e inspeccionarlo de forma voluntaria, pues la confrontación súbita y forzada con el estímulo novedoso induce, con mucha probabilidad, respuestas atemorizantes en los animales (Grandin \& Deesing, 2014).

El gamo (Dama dama) es una especie de cérvido endémica en la Península Ibérica mayormente en condiciones de libertad, aunque en las últimas décadas se ha puesto de manifiesto un interés por la domesticación y la cría de diferentes especies de cérvidos con diferentes grados de intensificación (Lista Mundial de Vigilancia para la Diversidad de los Animales Domésticos (2 ${ }^{\mathrm{a}}$ edición), Banco Global de Datos para la Diversidad de Recursos Genéticos de Animales de Granja, FAO) en granjas cinegéticas que producen ejemplares vivos para la repoblación de los terrenos de caza o la producción de piezas de carne para su comercialización (Silva et al., 2013). Las condiciones de estabulación propias de la cría intensiva de gamos en granjas cinegéticas generan en estos animales silvestres comportamientos anómalos y mortalidad por estrés, por lo que resulta fundamental considerar las particularidades biológicas y patrones conductuales en el medio natural de la familia Cervidae para minimizar los efectos perjudiciales de las labores de manejo de estos animales en granjas de producción, en las trabajos de atención sanitaria en centros de recuperación de fauna salvaje o en los programas de conservación de especies que se llevan a cabo en los parques zoológicos (Alderson, 2018).

El objetivo del presente estudio es caracterizar la respuesta neofóbica de un grupo de 8 gamos, mantenidos en cautividad, ante la presentación de un estímulo novel representado por cubos cebados de diferente color. Este hecho podrá ser de utilidad para un mejor entendimiento del papel que juegan la selección artificial o antrópica y los cambios en el ambiente sobre la conducta de animales silvestres, con el objetivo de lograr una optimización de las labores de manejo de estos animales en cautiverio y, por ende, de los índices de productividad.

\section{MATERIAL Y MÉTODOS}

\section{LOCALIZACIÓN Y MUESTRAS}

El experimento se llevó a cabo el 8 de junio de 2018 en las instalaciones del Zoológico de Córdoba (Córdoba, España). El estudio comenzó a las 8:30 h y se dio por finalizado a las 8:53 $\mathrm{h}$.

La población de animales objeto de estudio (8 gamos: 4 machos adultos, 1 vareto y 3 hembras; sin domesticar; de edades comprendidas entre 3,5 y 1,5 años) habita en un corral cercado (630 $\mathrm{m}^{2}$ aprox.) (Figura 1) con acceso a un corral anexo preparado para labores de manejo especializadas.

Como el rango de edad no estaba normalmente distribuido ( $\mathrm{P}<0,05$ tanto para el test de KolmogorovSmirnov como para el de Shapiro-Wilk's) utilizamos el mínimo, Q1, mediana, Q3 y máximo para describir el rango de edad en nuestra muestra. La edad mínima en el rango fue 1,50, el Q1 fue 2,50, la mediana fue 2,70, el Q3 fue 3,37 y la edad máxima fue 3,50.

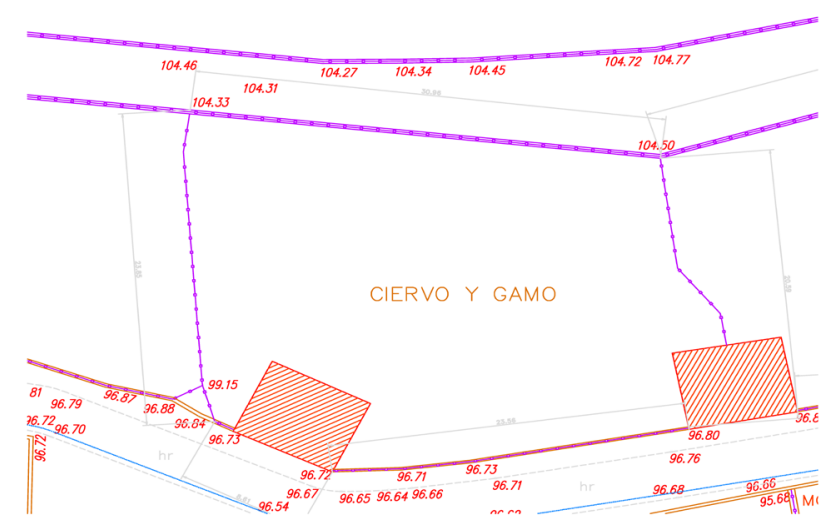

Figura 1. Plano de la instalación donde habita la población de animales objeto de estudio. Fuente: Zoológico de Córdoba (Map of the installation where the population of animals in the study inhabits). Fuente: Zoológico Municipal de Córdoba. 
ENCUESTA DE EVALUACIÓN CUALITATIVA DEL COMPORTAMIENTO (Qualtative BehaVIOUR Assessment, QBA)

De manera previa al test de neofobia, se evaluó cualitativamente la actitud de cada uno de los animales al inicio de cada una de las fases de este test por medio de una escala derivada de los resultados de una entrevista que se realizó a 10 individuos elegidos al azar, con el fin de evaluar la respuesta inicial de los animales frente a la presentación del estímulo problema, representado este por cinco cubos de diferentes colores.

El total de los entrevistados se componía de un $40 \%$ de cazadores, $20 \%$ veterinarios, $10 \%$ guardas forestales, $10 \%$ biólogos, $10 \%$ ingenieros agrónomos y $10 \%$ técnicos medioambientales. Las encuestas fueron realizadas desde el día 2 hasta el día 7 de junio de 2018.

El total de los entrevistados se componía de un $40 \%$ de cazadores, $20 \%$ veterinarios, $10 \%$ guardas forestales, $10 \%$ biólogos, $10 \%$ ingenieros agrónomos y $10 \%$ técnicos medioambientales.

La encuesta constaba de las preguntas reflejadas en la Tabla I.

En caso de empate entre las respuestas obtenidas, se primó la formación académica recibida en cuanto a las materias de etología y zoología, dada la implicación de este estudio con una especie animal silvestre, y para lo cual ciertos profesionales han recibido una mayor formación a lo largo de su trayectoria académica. El orden de importancia que se siguió de manera descendente con respecto a la formación de los encuestados

Tabla I. Relación de preguntas incluidas en la encuesta para la caracterización de la primera respuesta de los animales frente a la presentación del estímulo problema (List of questions included in the survey for the characterization of the first response of the animals against the presentation of the problem stimulus).

¿Cómo denominarías a un gamo que...

\begin{tabular}{|c|c|}
\hline Pregunta 1 & $\begin{array}{l}\text {...presta atención a otro estímulo externo y no al } \\
\text { estímulo de interés? }\end{array}$ \\
\hline Pregunta 2 & $\begin{array}{l}\text {...parece estar enfermo, decaído, apático, que } \\
\text { no responde a ningún estímulo? }\end{array}$ \\
\hline Pregunta 3 & $\begin{array}{l}\text {...presenta una postura normal y no presta } \\
\text { atención al estímulo de interés pero tampoco a } \\
\text { ningún otro estímulo externo? }\end{array}$ \\
\hline Pregunta 4 & $\begin{array}{l}\text {...presta atención tanto al estímulo de interés } \\
\text { como a otros estímulos externos? }\end{array}$ \\
\hline Pregunta 5 & $\begin{array}{l}\text {...presta atención sólo al estímulo de interés } \\
\text { pero no interacciona con este? }\end{array}$ \\
\hline Pregunta 6 & $\begin{array}{l}\text {...presta atención sólo al estímulo de interés y } \\
\text { mueve la cabeza alrededor de este? }\end{array}$ \\
\hline Pregunta 7 & $\begin{array}{l}\text {...presta atención sólo al estímulo de interés y se } \\
\text { mueve hacia el estímulo hasta quedarse cerca } \\
\text { de este? }\end{array}$ \\
\hline Pregunta 8 & $\begin{array}{l}\text {...presta atención sólo al estímulo de interés y se } \\
\text { acerca por completo a este? }\end{array}$ \\
\hline Pregunta 9 & $\begin{array}{l}\text {...presta atención sólo al estímulo de interés, se } \\
\text { acerca por completo a este e interacciona? }\end{array}$ \\
\hline Pregunta 10 & $\begin{array}{l}\text {...presta atención sólo al estímulo de interés e } \\
\text { intenta retirarse de este? }\end{array}$ \\
\hline
\end{tabular}

fue: biólogo, veterinario, ingeniero agrónomo, técnico medioambiental, guarda forestal y cazador.

\section{OtRAS CONSIDERACIONES PREVIAS}

Los animales no fueron separados del rebaño para proceder a su evaluación y participaron voluntariamente en la experiencia, ya que al estar tratando con animales silvestres la respuesta presentada podría verse condicionada por situaciones de estrés o ansiedad por separación. Para su identificación se emplearon las letras griegas $\alpha, \beta, \gamma, \delta$ y $\omega$, siguiendo los criterios de dominancia expuestos por Mech (1999) y diferenciando dos categorías en función del sexo.

El emplazamiento en el que tuvieron lugar las pruebas es la misma instalación en la que los animales desarrollan su actividad diaria, de modo que la única alteración presente en el ambiente fuese el estímulo novel representado por los cubos.

No se suministró alimento adicional a los animales fuera de su ración habitual. El alimento empleado para la experiencia fue siempre del mismo tipo y se dispensó de manera proporcional durante cada una de las fases del test de neofobia. La ración diaria constaba de $3.2 \mathrm{~kg}$ de pienso complementario Cervinanta 90 y $10 \mathrm{~kg}$ de zanahorias. Para las pruebas, se utilizó el total del pienso complementario de la ración diaria y tan sólo 5 $\mathrm{kg}$ de zanahorias. La primera fase empezó con $2.05 \mathrm{~kg}$ de alimento ( $0.8 \mathrm{~kg}$ de pienso y $1.25 \mathrm{~kg}$ de zanahorias). En fases sucesivas, para asegurar que todas las fases comenzaban con los $2.05 \mathrm{~kg}$ iniciales, se añadieron las cantidades correspondientes de pienso y zanahorias. El alimento sobrante tras la conclusión del test comportamental y que fue suministrado ad libitum fue $0.6 \mathrm{~kg}$ de pienso y $5 \mathrm{~kg}$ de zanahorias. La persona encargada de hacer los cambios necesarios de alimento y distribución de los cubos para pasar de una fase a otra, fue la persona a cargo de la alimentación de los animales de manera rutinaria.

Para la recogida de datos, se diseñaron hojas de campo específicas (Figura 2).

\section{TEST DE NEOFOBIA}

Los gamos fueron evaluados en un test de neofobia con el fin de caracterizar los posibles efectos condicionantes que pudieran derivarse de la presentación de los estímulos noveles representados por cubos de diferentes colores.

Para evaluar la respuesta neofóbica en estos animales se les presentaron cubos cebados de diferente color (negro ( $\mathrm{R}=36 ; \mathrm{G}=36 ; \mathrm{B}=36$; $\# 242424)$, gris $(\mathrm{R}=163$; $\mathrm{G}=173 ; \mathrm{B}=172$; \#A3ADAC), azul $(\mathrm{R}=0 ; \mathrm{G}=68 ; \mathrm{B}=254$; \#0044FE), rojo ( $R=254 ; G=67 ; B=76$; \#FE434C) y transparente) y se les concede un tiempo máximo de 300 segundos para que muestren interés por los cubos en cada una de las 4 fases que comprenden este test, a saber: fase 1 o cubos tapados, fase 2 o cubos tapados sólo un tercio de su superficie libre, fase 3 o cubos con tapa en posición invertida y comida encima de esta, y fase 4 o cubos tapados. Por mostrar interés se entiende que los animales presten atención, se acerquen o interaccionen con los cubos. El tiempo de reposo entre una fase y otra es de 60 segundos. 


\section{TEST DE NEOFOBIA}

Animal:

Valorador:

Fecha:

Para caracterizar la neofobia de un animal, cuando se le presentó un nuevo dispositivo (cubos tapados y cebados de diferente color: negro $(R=36 ; G=36 ; B=36 ; \# 242424)$, gris $(R=163 ; G=173$; $\mathrm{B}=172$; \#A3ADAC), azul $(\mathrm{R}=0 ; \mathrm{G}=68 ; \mathrm{B}=254$; \#0044FE), rojo $(\mathrm{R}=254 ; \mathrm{G}=67 ; \mathrm{B}=76$; \#FE434C) y transparente), se determinó el tiempo que:

\begin{tabular}{l|l|l|l|l|}
\cline { 2 - 4 } & $\begin{array}{c}\text { Cubos tapados, el } \\
\text { animal desconoce } \\
\text { que están cebados }\end{array}$ & $\begin{array}{c}\text { Cubos } \\
\text { destapados }\end{array}$ & $\begin{array}{c}\text { Cubos tapados } \\
\text { sólo 1/3 de su } \\
\text { superficie libre }\end{array}$ & $\begin{array}{c}\text { Cubos tapados, el } \\
\text { animal sabe que } \\
\text { están cebados }\end{array}$ \\
\hline $\begin{array}{l}\text { El animal está distraído, sin } \\
\text { prestar atención al estímulo de } \\
\text { interés pero sí a otros estímulos } \\
\text { externos }\end{array}$ & & & & \\
\hline $\begin{array}{l}\text { El animal tarda en prestar } \\
\text { atención al estímulo de interés }\end{array}$ & & & & \\
\hline $\begin{array}{l}\text { El animal permanece inmóvil e } \\
\text { intenta aproximarse con la } \\
\text { cabeza hacia el estímulo de } \\
\text { interés }\end{array}$ & & & & \\
\hline $\begin{array}{l}\text { El animal tarda en aproximarse } \\
\text { al estímulo de interés }\end{array}$ & & & & \\
\hline $\begin{array}{l}\text { El animal tarda en aproximarse } \\
\text { por completo al estímulo de } \\
\text { interés }\end{array}$ & & & & \\
\hline $\begin{array}{l}\text { El animal tarda en intentar } \\
\text { destapar alguno de los cubos }\end{array}$ & & & & \\
\hline
\end{tabular}

La disposición de los cubos será la siguiente:
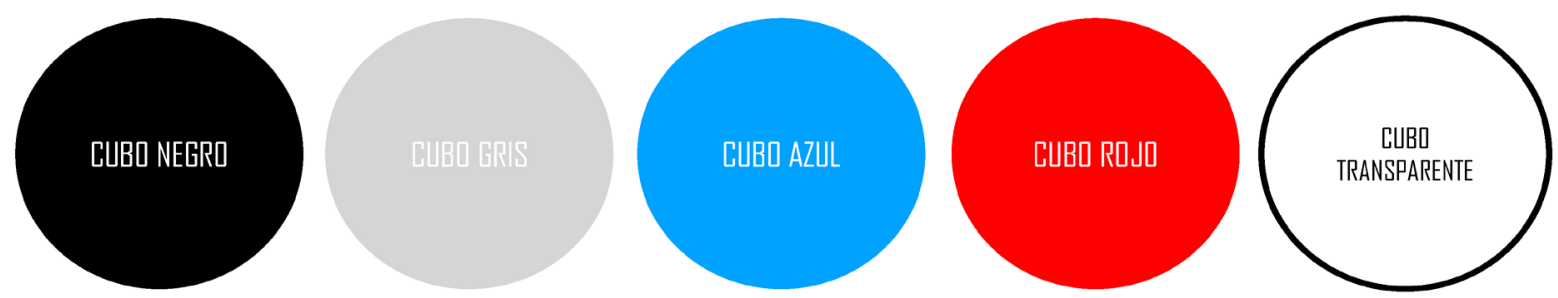

Figura 2. Hoja de campo para la recogida de datos (Data collection field sheet).

Para cada una de las cuatro fases del test se midió: el tiempo que el animal estaba distraído, sin prestar atención al estímulo novel pero sí a otros estímulos externos; el tiempo que el animal tardaba en prestar atención al estímulo de interés pero permanecía inmóvil; el tiempo que el animal tardaba en aproximarse al estímulo de interés a un metro de distancia; el tiempo que el animal tardaba en tocar el estímulo novel de manera intencionada con cualquier parte de su cuerpo; y el tiempo que el animal tardaba en intentar acceder a la comida de alguno de los cubos.

Para el experimento se usaron cubos cilíndricos de plástico de diferentes colores (negro $(R=36 ; G=36$;
$B=36$; $\# 242424)$, gris $(R=163 ; G=173 ; B=172$; \#A3ADAC), azul $(\mathrm{R}=0 ; \mathrm{G}=68 ; \mathrm{B}=254 ; \# 0044 \mathrm{FE})$ y rojo $(\mathrm{R}=254 ; \mathrm{G}=67$; $\mathrm{B}=76$; \#FE434C)) de $38.5 \mathrm{~cm}$ de diámetro y $57.6 \mathrm{~cm}$ de alto; y un cubo cilíndrico transparente $(\varnothing=38.6 \mathrm{~cm} \mathrm{y}$ $70.2 \mathrm{~cm}$ alto). Para la determinación de la composición en los canales primarios (azul, rojo y verde) de cada uno de los colores y tonos empleados en las pruebas, se utilizó la aplicación ColorMeter Free versión 3.0.0.

Todos los ensayos fueron filmados con dos cámaras (30 fotogramas/segundo) situadas a $3.716 \mathrm{~m}$ de los cubos, en dos localizaciones diferentes del recinto, para poder evaluar las puntuaciones obtenidas tras la conclusión del test comportamental. 


\section{DesARROLLO DEL TEST}

La duración total del test completo, desde que se presentan los cubos tapados en la primera fase hasta su retirada tras la conclusión de la cuarta y última fase, es de 1380 segundos. En ningún caso se concedió tiempo adicional para que los gamos reaccionaran ante el estímulo novel representado por los cubos. La pausa entre las diferentes fases fue de 60 segundos.

El hecho de que un animal concluyese satisfactoriamente las pruebas, entendido esto como que el animal consigue acceder a la comida de alguno de los cinco cubos, no condicionó la retirada del estímulo presentado hasta que el tiempo determinado para cada una de ellas hubiese transcurrido por completo. La Figura 3 presenta esquemáticamente el desarrollo de la prueba

\section{REGISTRO DE OBSERVACIONES}

Tras revisión bibliográfica, se determinó la toma de valores para cada animal para las variables correspondientes a:

Edad: para la determinación de la edad de los animales se tomaron en cuenta los criterios expuestos por Cabrera (1914) y Bartoš and Losos (1997). Como el rango de edad no estaba normalmente distribuido $(\mathrm{P}<0,05$ tanto para el test de Kolmogorov-Smirnov como para el de Shapiro-Wilk's) utilizamos el mínimo, $\mathrm{Q}_{1}$, mediana, $\mathrm{Q}_{3}$ y máximo para describir el rango de edad en nuestra muestra. La edad mínima en el rango fue 1,50, el $Q_{1}$ fue 2,50, la mediana fue 2,70, el $Q_{3}$ fue 3,37 y la edad máxima fue 3,50.

Desarrollo de la cuerna: se determinó tomando en cuenta tanto el cronograma expuesto por Cabrera (1914) como los periodos de desarrollo de las cuernas descritos por Bartoš y Losos (1997). Esta variable se midió empleando una escala ordinal. Clasificamos el desarrollo de las cuernas de los animales incluidos en nuestro estudio en cuatro niveles. El primer nivel representa a animales acornes (hembras y jóvenes menores de 1 año). El nivel 2 coincide con el periodo de crecimiento 1 descrito por Bartoš and Losos (1997) y podría atribuirse a machos varetos que se encuentran en el rango de edad de 1 a 1,5 años, de acuerdo a Cabrera (1914). El nivel 3, coincide con aquellos animales que se encuentran en los periodos de desarrollo 2 y 3 descritos por Bartoš and Losos (1997), correspondientes al rango de edad comprendido entre 2 a 2,5 años. El nivel 4, se corresponde con el cuarto periodo de crecimiento de Bartoš and Losos (1997) y fue atribuído a animales cuya edad se situaba en torno a los 3-4 años. Categorías superiores no fueron contempladas debido a que no encontramos estadíos de desarrollo más avanzados en nuestra muestra.

Peso vivo: se estimó aplicando el cuadro de equivalencia entre el diámetro torácico y el peso vivo publicado por el Pennsylvania State University Department of Dairy and Animal Science y The Pennsylvania Game Commission (2018) (http:/ /www.pgc.pa.gov). El diámetro costal fue calculado con la ayuda de una pica calibrada de $2 \mathrm{~m}$ con marcas bicolores (rojo/blanco) de $10 \mathrm{~cm}$ cada una, situada junto al comedero, en una superficie nivelada.

Jerarquía: para la identificación de los animales se emplearon las letras griegas $\alpha, \beta, \gamma, \delta$ y $\omega$, siguiendo los criterios de dominancia y diferenciando dos jerarquías separadas en función del sexo tal y como describe (Mech (1999)) para los animales en cautividad. Por tanto, consideramos la jerarquía como una variable categórica compuesta por 5 niveles.

Sexo: variable dicotómica con dos niveles, 1 para los machos y 2 para las hembras.

Actitud del animal: la actitud del animal fue valorada en los primeros 10 segundos desde el comienzo de la prueba siguiendo una escala categórica con 10 niveles clasificando el grado de aversión frente al estímulo presentado de manera creciente. En 10 segundos puede darse un cambio de actitud en el animal en varias ocasiones. Sin embargo, con esta variable lo que pretendíamos era describir cualitativamente la primera reacción puesta en práctica por los animales frente a la presentación del estímulo novel en cada una de las fases del test.

Tiempo: tiempo que el animal está distraído, sin prestar atención al estímulo novel pero sí a otros estímulos externos; tiempo que el animal tarda en prestar atención al estímulo de interés pero permanece inmóvil; tiempo que el animal tarda en aproximarse al estímulo de interés a un metro de distancia; tiempo que el animal tarda en tocar el estímulo novel de manera intencionada con cualquier parte de su cuerpo; y tiempo que el animal tarda en intentar acceder a la comida de alguno de los cubos. Se tomó con la ayuda de un cronómetro.

\section{SISTEMA DE EVALUACIÓN DE LAS PRUEBAS}

La evaluación de cada una de las fases se llevó a cabo por cuatro valoradores diferentes entrenados a
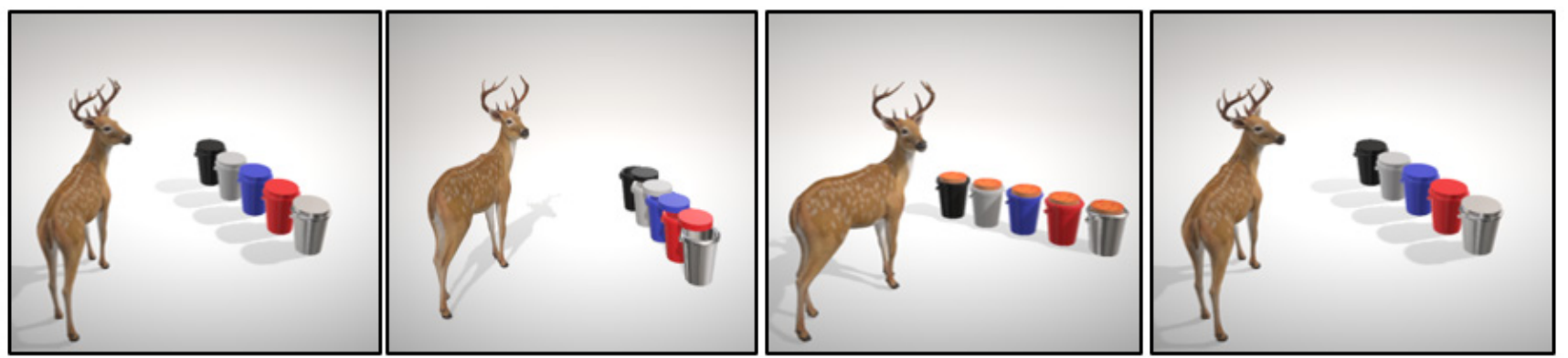

Figura 3. Representación gráfica y secuencial de las cuatro fases del test de neofobia (Graphic and sequential representation of the four phases of the Neophobia test). 
tal fin, bajo condiciones idénticas, una vez concluido el experimento y sobre el material gráfico recogido durante las experiencia de campo.

\section{ESTUDIO ESTADÍSTICO}

\section{FIABILIDAD DE LA ESCALA DE MEDIDA INTRA E INTEROBSERVADOR}

Para testar la fiabilidad interobservador se compararon las medidas de cada par de valoradores contemplando todas las combinaciones posibles. Del mismo modo, la fiabilidad intraobservador se llevó a cabo comparando las medidas tomadas por el mismo valorador durante las dos valoraciones efectuadas. En ambos casos empleamos el test de la kappa de Cohen del procedimiento Tablas Cruzadas de SPSS Statistics para Windows, Version 24.0, IBM Corp. (2016).

Se calcularon los intervalos de confianza al $95 \%$ para cada una de las posibles combinaciones inter e intraobservador. El intervalo de confianza fue calculado de acuerdo a la siguiente expresión: 95\% kappa $\mathrm{IC}=\kappa \pm 1.96 \mathrm{SE} \kappa$, donde: $\mathrm{SE} \kappa=[(\mathrm{po}(1-\mathrm{po}) / \mathrm{n}(1-\mathrm{pe}) 2] 0.5$; con el procedimiento de Tablas Cruzadas de SPSS Statistics para Windows, Version 24.0, IBM Corp. (2016).

TEST DE MULTICOLINEALIDAD ENTRE VARIABLES CONTINUAS Y VARIABLES CONTINUAS Y NOMINALES

Como paso previo a la reducción de la dimensionalidad de un conjunto de variables debemos cerciorarnos de que no existe multicolinealidad entre las variables que queremos medir. Esto quiere decir que no exista relación lineal directa entre ellas. La medida de asociación cuantifica la fuerza de relación entre dos variables. El índice de asociación d de Cohen mide la relación entre una variable categórica dicotómica (sexo) y una variable cuantitativa. Los valores que puede tomar d no están acotados a un rango, pudiendo ser tanto positivos como negativos. Si las dos variables consideradas son independientes entonces d será igual a 0 , mientras que cuanto mayor sea la asociación entre ellas, mayor será el valor de d en términos absolutos. Cohen sugiere las siguientes normas interpretativas, aunque el propio autor afirma que se deben utilizar sólo en el caso que no se tenga ningún criterio sustantivo que sirva de base de interpretación: valores absolutos de d entre 0,2 y 0,5 indicarían una intensidad de la asociación (tamaño del efecto) baja; entre 0,5 y 0,8, media; mientras que a partir de 0,8 , alta.

Por otro lado, el índice f de Cohen permite analizar la relación entre una variable cuantitativa y una categórica en el caso en que esta última tenga más de dos niveles posibles (k valores). Se basa para ello en el cálculo de la dispersión de las medias de los diferentes subgrupos definidos por los $\mathrm{k}$ niveles de la variable categórica o nominal. El valor de la f de Cohen será siempre mayor o igual a 0 , tanto mayor cuanto más intensa sea la asociación entre las variables.

SPSS no puede calcular el valor f o d de Cohen directamente, pero sí pueden calcularse mediante $E t a^{2}$ parcial $\left(\eta p^{2}\right)$. Cohen analiza la relación entre los valores f de Cohen y Eta $a^{2}$ parcial:

$$
\eta^{2}=\frac{f^{2}}{\left(1+f^{2}\right)} \quad ; \quad f=\sqrt{\frac{\eta^{2}}{\left(1-\eta^{2}\right)}}
$$

Donde $\mathrm{f}^{2}$ es el cuadrado del tamaño del efecto, y $\eta^{2}$ es el $\eta p^{2}$ calculado por SPSS (Cohen, 1988).

Por su parte, el coeficiente d de Cohen se calcula mediante la siguiente fórmula:

$$
\mathrm{d}=\frac{M_{1}-M_{2}}{\sqrt{\frac{S_{1}^{2}+S_{2}^{2}}{2}}} \quad ; \quad \mathrm{r}=\frac{d}{\sqrt{d^{2}+4}}
$$

Donde $\mathrm{d}$ es el índice de Cohen, $\mathrm{M}_{1}$ es la media del primer grupo, $M_{2}$ la media del segundo grupo, $S_{1}$ la desviación típica del primer grupo, $S_{2}$ la desviación típica del segundo grupo y r el coeficiente del tamaño del efecto.

Del mismo modo, empleamos el coeficiente de correlación de Pearson para medir la relación entre pares de variables medidas a nivel continuo.

El análisis de correlaciones de Pearson se llevó a cabo con el procedimiento Correlaciones de SPSS Statistics para Windows, Version 24.0, IBM Corp. (2016), mientras que y $\eta p^{2}$ para el cálculo posterior de los coeficientes d y f de Cohen fueron calculados con el procedimiento Tablas Cruzadas del mismo paquete estadístico.

\section{REDUCCIÓN DE LA DIMENSIONALIDAD DE LAS VARIABLES}

El análisis de componentes principales categóricos (CATPCA) es una técnica multivariada apropiada para reducir la dimensionalidad de un conjunto de datos procedente de un gran número de variables interrelacionadas, mientras se mantiene tanto como se puede la variación presente en este conjunto de datos.

Esto se consigue transformando este conjunto de datos en un nuevo set de variables, los componentes principales (PCs), no correlacionados, y ordenados de tal forma que los primeros retengan la mayor parte de la variación presente en las variables originales. Al contrario que el análisis de componentes principales tradicional, que asume relaciones lineales entre variables numéricas (continuas o discontinuas), el CATPCA, por medio del enfoque del escalamiento óptimo, permite que las variables estén escaladas a diferentes niveles. Por tanto, las variables categóricas pueden ser cuantificadas dentro de una dimensionalidad predefinida. Como resultado, las posibles relaciones no lineales entre variables pueden ser modeladas.

No se llevó a cabo un estudio de regresión categórica sobre el test de neofobia dado que el fin de esta prueba solo era el análisis preliminar de las variables consideradas.

\section{RESULTADOS Y DISCUSIÓN}

La escala de evalación cualitativa del comportamiento fue obtenida al parametrizar numéricamente los adjetivos calificativos resultantes de la encuesta preliminar de valoración de la axtitud de los animales. Los valores numéricos que se atribuyeron a los adjetivos calificativos tomaron en cuenta la actitud de los gamos con respecto al estímulo presentado, siendo 1 un animal que presta atención a otro estímulo externo 
y no al estímulo de interés, y 10 un animal que presta atención sólo al estímulo de interés e intenta retirarse de este.

La primera precaución a considerar antes de comenzar un estudio sobre el comportamiento de animales silvestres son los efectos de la presencia del observador sobre el comportamiento. En esta situación es fundamental servirse de aparatos que ayuden a realizar observaciones a distancia. La observación en proximidad requiere del uso de escondites a menos que los animales estén habituados a la presencia humana, como es nuestro caso, donde los gamos del zoológico están habituados a la presencia humana.

El experimento completo fue valorado en dos ocasiones por cuatro valoradores y se testó para comprobar si existía concordancia intra e interobservador con el mismo procedimiento. En este caso, el test de la kappa de Cohen demostró que la concordancia interobservador (de 0.753 a $0.759 \mathrm{P}<0.001$ ) e intraobservador (de 0.629 a $0.686 ; \mathrm{P}<0.001$ ) era sustancial. Esto sugiere que la escala utilizada y los métodos empleados para entrenar a los valoradores fueron lo suficientemente sólidos, eficaces y fiables. Esto sugiere que la escala utilizada y los métodos empleados para entrenar a los valoradores fueron lo suficientemente sólidos, eficaces y fiables.

Para el test de neofobia, se detectó que las variables estaban desde pobremente correlacionadas hasta altamente correlacionadas, tanto positiva como negativamente. Las correlaciones negativas entre las variables medidas variaron desde -0,005 (entre el tiempo que el animal está distraído y la edad) hasta $-0,912$ (entre la edad y la jerarquía), y las positivas desde 0,005 (entre el tiempo de aproximación a un metro y la edad) hasta 0,831 (entre el tiempo que el animal está distraído y el tiempo que el animal tarda en prestar atención, pero permanece inmóvil).

El análisis de componentes principales categórico reveló que la variabilidad presente en la población para la prueba de neofobia explicaba un $69.94 \%$ de la varianza, con dos dimensiones (componente principal $1=0,877$, componente principal $2=0,714$ ). Las variables actitud $(0,792)$, tiempo que animal está distraído $(-0,734)$, tiempo que tarda en prestar atención pero permanece inmóvil $(-0,682)$, tiempo que tarda en aproximarse al estímulo a un metro $(-0,782)$, tiempo que tarda en tocar el cubo $(-0,772)$, tiempo que tarda en acceder a la comida $(-0,509)$, desarrollo de la cuerna $(0,750)$ y peso vivo $(0,834)$, presentaron unos componentes de carga superiores a 0,5 en valor absoluto $y$, por tanto, fueron retenidos en el modelo para la dimensión 1. La dimensión 2, sin embargo, presentó componentes de carga superiores a 0,5 en valor absoluto para las variables sexo $(0,590)$, jerarquía $(-0,947)$, edad $(0,967)$ y grado de desarrollo de la cuerna $(-0,590)$.

El estudio de los efectos de la domesticación y del manejo sobre el comportamiento podrían servir como punto de referencia para comparar los patrones de comportamiento de los miembros domésticos y salvajes de la misma especie. Así, podrían no solo mejorarse las prácticas de manejo de los animales confinados sino también diseñar experiencias que comparasen las consecuencias de la cría en confinamiento durante varias generaciones de una especie silvestre con la cría en idénticas condiciones ambientales de otra especie doméstica muy estrechamente relacionada.

\section{CONCLUSIÓN}

El conocimiento del comportamiento animal también es necesario para proporcionarles la dieta, el alojamiento y la convivencia social más adecuada a aquellos que se crían en un entorno humano. Además, por nuestra propia seguridad, debemos reconocer la agresión, el miedo y otras emociones de los animales, y emplear estos conocimientos a la hora de calmar o sujetar a los animales de manera eficiente cuando sea necesario. La relación estrés-enfermedad tiene un elevado impacto económico y manifiesta de manera muy directa la importancia del comportamiento animal en la medicina veterinaria. Existe también una relación directa entre el rango en escala social y el nivel inmunológico del individuo. El comportamiento animal preside muchos aspectos de la zootecnica por cuatro razones básicas: para criar y producir, para incrementar la productividad, para estimar la heredabilidad de determinados comportamientos y para optimizar la mecanización de las explotaciones. Para el manejo de animales silvestres que se mantienen en cautiverio, se deben seleccionar los animales por determinados comportamientos deseables, al igual que por sus características físicas y aptitudes para la producción. En animales de granja es muy importante la docilidad en el manejo, el comportamiento maternal y la capacidad de adaptación a los sistemas de cría y alimentación en grupo, entre otras características. La mayor parte de las investigaciones actuales en etología aplicada están enfocadas a lograr un mayor entendimiento de la interacción de los animales bajo ambientes extremadamente artificiales.

\section{BIBLIOGRAFÍA}

Alderson, G 2018, 'Conservación de razas y mantenimiento de la biodiversidad: justificación y metodología para la conservación de los recursos genéticos animales', Archivos de Zootécnia, vol. 67, no. 258 , pp. 300-9.

Bartoš, L \& Losos, S 1997, 'Response of antler growth to changing rank of fallow deer buck during the velvet period', Canadian Journal of Zoology, vol. 75, no. 11, pp. 1934-9.

Cabrera, A 1914, Fauna Ibérica. Mamíferos. Museo Nacional de Ciencias Naturales, Madrid. 441 pp, Spanish.

Cohen, J 1988, Statistical power analysis for the behavioral sciences. 2nd, Hillsdale, NJ: erlbaum.

Commission, PSUDoDaASaTPG 2018, Deer Weight Estimating Chart, viewed 01/06/2018, <http://www.pgc.pa.gov/Wildlife/WildlifeSpecies/White-tailedDeer/Pages/DeerWeightChart.aspx>.

Coolen, I, Bergen, YV, Day, RL \& Laland, KN 2003, 'Species difference in adaptive use of public information in sticklebacks', Proceedings of the Royal Society of London B: Biological Sciences, vol. 270, no. 1531, pp. 2413-9.

Dall, SR, Giraldeau, L-A, Olsson, O, McNamara, JM \& Stephens, DW 2005, 'Information and its use by animals in evolutionary ecology', Trends in ecology \& evolution, vol. 20, no. 4, pp. 187-93.

Denis, B 2004, 'La domestication: un concept devenu pluriel', Productions Animales 3 (17), 161-166.(2004). 
Grandin, T \& Deesing, MJ 2014, 'Behavioral genetics and animal science', in Genetics and the Behavior of Domestic Animals (Second Edition), Elsevier, pp. 1-40.

Gross, BL \& Olsen, KM 2010, 'Genetic perspectives on crop domestication', Trends in plant science, vol. 15, no. 9, pp. 529-37.

Larson, G, Piperno, DR, Allaby, RG, Purugganan, MD, Andersson, L, Arroyo-Kalin, M, Barton, L, Vigueira, CC, Denham, T \& Dobney, K 2014, 'Current perspectives and the future of domestication studies', Proceedings of the National Academy of Sciences, p. 201323964.

Mech, LD 1999, 'Alpha status, dominance, and division of labor in wolf packs', Canadian Journal of Zoology, vol. 77, no. 8, pp. 1196-203.

Mettke区Hofmann, C, Winkler, H \& Leisler, B 2002, 'The significance of ecological factors for exploration and neophobia in parrots', Ethology, vol. 108, no. 3, pp. 249-72.

Schultz, DP 2013, Sensory restriction: Effects on behavior, Elsevier.
Silva, MCd, Lopes, FB, Paulini, F, Fioravanti, MCS, McManus, CM, Félix, GA \& Sereno, JRB 2013, 'Participação e empoderamento: princípios para a conservação de recursos zoogenéticos on farm', Archivos de Zootécnia, vol. 62, no. 237, pp. 93-104.

Sol, D, Timmermans, S \& Lefebvre, L 2002, 'Behavioural flexibility and invasion success in birds', Animal Behaviour, vol. 63, no. 3, pp. 495-502.

Stöwe, M, Bugnyar, T, Heinrich, B \& Kotrschal, K 2006, 'Effects of group size on approach to novel objects in ravens (Corvus corax)', Ethology, vol. 112, no. 11, pp. 1079-88.

Stöwe, M, Bugnyar, T, Loretto, M-C, Schloegl, C, Range, F \& Kotrschal, K 2006, 'Novel object exploration in ravens (Corvus corax): effects of social relationships', Behavioural Processes, vol. 73, no. 1, pp. 68-75.

Swaney, W, Kendal, J, Capon, H, Brown, C \& Laland, KN 2001, 'Familiarity facilitates social learning of foraging behaviour in the guppy', Animal Behaviour, vol. 62, no. 3, pp. 591-8. 